\title{
Arteriolopatía urémica calcificante: presentación de caso clínico con manejo médico conservador
}

\author{
Calcific uremic arteriolopathy: presentation of a clinical case with conservative medical management
}

\author{
Mónica Marcela Macías Ortega
}

Médico Especialista en Medicina Interna. Actualmente Médico-residente del Segundo Año de la subespecialidad de Endocrinología (2019-2021), Centro Médico Nacional 20 de Noviembre, Instituto de Seguridad Social de los Trabajadores del Estado (ISSSTE), Ciudad de México, México.

RESUMEN. Antecedentes: La arteriolopatia calcificante urémica o calcifilaxis es un síndrome raro, potencialmente mortal, que afecta casi en exclusiva a pacientes con insuficiencia renal y diálisis, caracterizado por calcificación vascular de arterias de pequeño y mediano calibre, con posterior proliferación, fibrosis y trombosis que conducen finalmente a necrosis y úlceras cutáneas. Se asocia con la enfermedad renal crónica terminal y trasplante renal, con prevalencia de $1-4 \%$ de los pacientes con insuficiencia renal crónica. El tratamiento es especializado a base de cámara hiperbárica y paratiroidectomía para inducir curación. Descripción del caso clínico: Femenina de 42 años, captada en la consulta externa de nefrología en el Instituto Hondureño de Seguridad Social en el año 2017, con antecedente de hipertensión arterial y nefropatía crónica, sometida a trasplante renal en 1998 el cual fue fallido, posteriormente en programa de hemodiálisis y manejo conservador desde el año 2005. La paciente desarrolló lesiones equimóticas en tronco y úlceras en sitios de nódulos subcutáneos que se sobreinfectaron, desarrollando signos de respuesta inflamatoria sistémica. Los exámenes de laboratorio mostraron hiperfosfatemia, paratohormona $3518 \mathrm{pg} / \mathrm{ml}$, producto calcio-fósforo 73.5. Ante la falta de manejo quirúrgico (paratiroidectomía) y cámara hiperbárica en la institución, en el 2017 se estableció tratamiento conservador a base de antibióticos, analgésicos, y hemodiálisis diarias, con lo que presentó mejoría del cuadro clínico; sin embargo, sin resolución de su cuadro de base de la calcifilaxis. Conclusión: El manejo conservador en el caso de pacientes con calcifilaxis es una opción de tratamiento disponible con buena respuesta en pacientes con seguimiento estrecho.

Palabras claves: Calcificación vascular, Hiperparatiroidismo secundario, Insuficiencia renal, Uremia.

Recibido: 26-06-2019 Aceptado para publicación 26-11-2020

Dirección para correspondencia: Dra. Mónica Marcela Macías Ortega

Correo electrónico: ortega.3mmm @gmail.com

Declaración de relaciones y actividades financieras y no financieras y conflictos de interés: ninguno.

DOI: https://doi.org/10.5377/rmh.v88i2.11493

\section{INTRODUCCIÓN}

La arteriolopatía calcificante urémica o calcifilaxis es producida por una calcificación sistémica de la túnica media de las arteriolas que da lugar a isquemia y a necrosis subcutánea. ${ }^{1-3}$ Se asocia con enfermedad renal crónica terminal y trasplante renal. Su prevalencia es $1-4 \%$ en pacientes con enfermedad renal crónica (ERC). La mortalidad es alta por las complicaciones derivadas de la infección de lesiones en piel, si las lesiones son distales tienen mortalidad de $32 \%$ y proximales de $63 \%$. La patogenia se desarrolla en medio urémico relacionada a hiperparatiroidismo, hiperfosforemia, tratamientos que producen hipercalcemia, como la vitamina $D$ y quelantes del fósforo., ${ }^{1,2}$ Otros factores incluidos en la patogenia son: la deficiencia de inhibidores de la calcificación vascular como la fetuína $A$ y la proteína Gla, la obesidad, el sexo femenino, la diabetes, hipoalbuminemia y estados de hipercoagulabilidad por deficiencia de proteínas $\mathrm{C}$ y $\mathrm{S}$ o con anticoagulantes orales. ${ }^{2-4}$

En relación a la fisiopatología, en la ERC se disminuye el aclaramiento renal de fósforo, disminuye la síntesis de 1,25-dihidroxicolecalciferol a partir de 25-colecalciferol, disminuye la absorción intestinal de calcio con hipocalcemia lo que estimula la producción de hormona paratiroidea (PTH) ocasionando hiperparatiroidismo secundario. La prevalencia de enfermedad adinámica del hueso en pacientes en diálisis es del 15 al 60\%; varios estudios sugieren incremento de su incidencia por introducción de calcio en la diálisis, que contiene aglutinantes de fosfatos como agentes terapéuticos para la hiperfosfatemia severa. ${ }^{4-7}$ Las alteraciones del metabolismo fosfocálcico favorecen el depósito vascular de calcio (Ca+)., ${ }^{5,6}$ Se debe hacer diagnóstico diferencial con vasculitis sistémicas, arterioesclerosis periférica, síndrome antifosfolípido, necrosis cumarínica y endocarditis infecciosa. ${ }^{3-5}$

Hay dos formas diferentes de presentación clínica. La proximal abarca tronco, abdomen, glúteos y muslos. La forma acra (distal) compromete extremidades y el pene. Ambas pueden estar presentes en un mismo paciente. ${ }^{2,4}$ Los diferentes estudios relacionan la calcifilaxis proximal con los pacientes diabéticos, siendo las alteraciones del metabolismo fosfocálcico 
más severas que en los pacientes con calcifilaxis acra y suelen tener peor pronóstico..$^{3-6}$ Algunos desencadenantes son trauma local, empleo de corticosteroides, sales de hierro, sales metálicas inorgánicas, proteínas de origen animal, la polixina, la 5-hidroxitriptamina, y otros degranuladores mastocitarios, y medios de contraste. La hipertensión arterial y la alcalosis metabólica post hemodiálisis podrían predisponer al desarrollo de calcifilaxis. ${ }^{4,6}$

El tratamiento se basa en anular factores implicados en su aparición, inhibir la inflamación y los depósitos de calcio. En la curación intensiva de las úlceras se incluye el sistema Vacuum Assisted Closure (VAC), la cámara hiperbárica y tratamiento de las lesiones, analgesia y promoción del buen estado nutricional. ${ }^{4-8}$

Entre el manejo médico conservador disponible se encuentra la hemodiálisis diaria, el manejo de la uremia, los quelantes del fósforo, analgésicos y antibióticos con curaciones para las lesiones en piel. No incluye la cámara hiperbárica ni paratiroidectomía en todos los casos. En Honduras existe poca información acerca de la evolución y opciones de tratamiento para la calcifilaxis. Por lo tanto, es necesaria la descripción de casos que permitan conocer la evolución clínica y opciones de manejo conservador disponibles en el país y su pronóstico a mediano plazo.

\section{DESCRIPCIÓN DEL CASO CLÍNICO}

Paciente femenina de 42 años de edad, obesa, con antecedente de glomerulopatía de etiología indeterminada e hipertensión arterial secundaria; en control en la Consulta Externa de Nefrología en el año 2017 en el Instituto Hondureño de Seguridad Social (IHSS), Tegucigalpa. En 1998 fue sometida a trasplante renal con buena funcionalidad del trasplante por 7 años y posteriormente con rechazo de éste por la falta de apego al tratamiento inmunosupresor (azatioprina, prednisona y ciclosporina).

Se inicia en programa de hemodiálisis en año 2005 por fistula arteriovenosa y catéter temporal. Se le realizaron sesiones con duración de 4 horas, tres veces a la semana hasta el año 2015. En ese año, se sometió a diálisis peritoneal por agotamiento de acceso vascular, durante 7 meses, hasta la colocación de injerto politetrafluroetileno (PTFe). En el año
2018 continúa con hemodiálisis tres veces a la semana con una duración de 4 horas por sesión.

Se realizaron exámenes de laboratorio trimestralmente, en enero del 2016 los resultados de química sanguínea demostraron los siguientes valores: hormona paratiroidea (PTH) 3776.50 $\mathrm{pg} / \mathrm{ml}$, calcio $9.6 \mathrm{mg} / \mathrm{dl}$, fósforo $6.7 \mathrm{mg} / \mathrm{dl}$, hemoglobina $(\mathrm{Hb})$ $9.3 \mathrm{~g} / \mathrm{dl}$, hematocrito (HTC) $28.2 \%$, plaquetas $269,000 /$ microlitro, leucocitos 5,400 mm3/microlitro, ferritina $1500 \mathrm{ng} / \mathrm{ml}$, hierro sérico121 microgramo/dl. La paciente recibía tratamiento con paracalcitol 37 microgramos a la semana y eritropoyetina (EPO) 2000 unidades internacionales (UI) subcutáneas 6 veces a la semana (12,000 UI/semana).

La paciente se presentó en Consulta Externa de Nefrología del IHSS en marzo del 2017, refiriendo dolor en tórax anterior y en abdomen. El dolor se intensificó durante la realización de su hemodiálisis y se mantuvo durante todo el procedimiento, no se atenuó con analgésicos, antiinflamatorios no esteroideos (AINES) ni con fármacos opioides. Al examen físico se encontró con presión arterial 120/70 mmHg, frecuencia cardiaca y pulso 90 por minuto, frecuencia respiratoria 17 por minuto; biotipo pícnico, crónicamente enferma, luciendo mayor que su edad cronológica. A la inspección se identificaron lesiones maculares violáceas en tórax anterior y muslos. También lesiones nodulares dolorosas de aproximadamente $3 \mathrm{~cm}$ diseminadas en tórax, abdomen y muslos. Además, se palparon calcificaciones en el tejido subcutáneo abdominal, algunas ulceradas de forma superficial en lesiones eritematovioláceas en el tórax anterior y una úlcera profunda en hemiabdomen derecho, con supuración, sangrado y cambios inflamatorios alrededor (Figura 1).

En mayo de 2017 presenta resultados de química sanguínea con PTH $3518 \mathrm{pg} / \mathrm{ml}$, calcio $9.8 \mathrm{mg} / \mathrm{dl}$, fósforo 7.5 $\mathrm{mg} / \mathrm{dl}$ y producto calcio-fósforo: 73.5 , que sugieren la presencia de hiperparatiroidismo terciario. Además, hemoglobina $8.8 \mathrm{~g} /$ $\mathrm{dl}$, hematocrito $27.5 \%$, plaquetas $270,000 /$ microlitro, leucocitos $5,700 \mathrm{~mm} 3 /$ microlitro, ferritina $1860 \mathrm{ng} / \mathrm{ml}$, hierro sérico 84 microgramo/dl. Se diagnostica clínicamente con calcifilaxis e hiperparatiroidismo terciario. Se inicia tratamiento conservador con sevelamero 2 tabletas cada semana, eritropoyetina 12,000 UI a la semana, morfina $5 \mathrm{mg}$ subcutánea post-hemodiálisis y
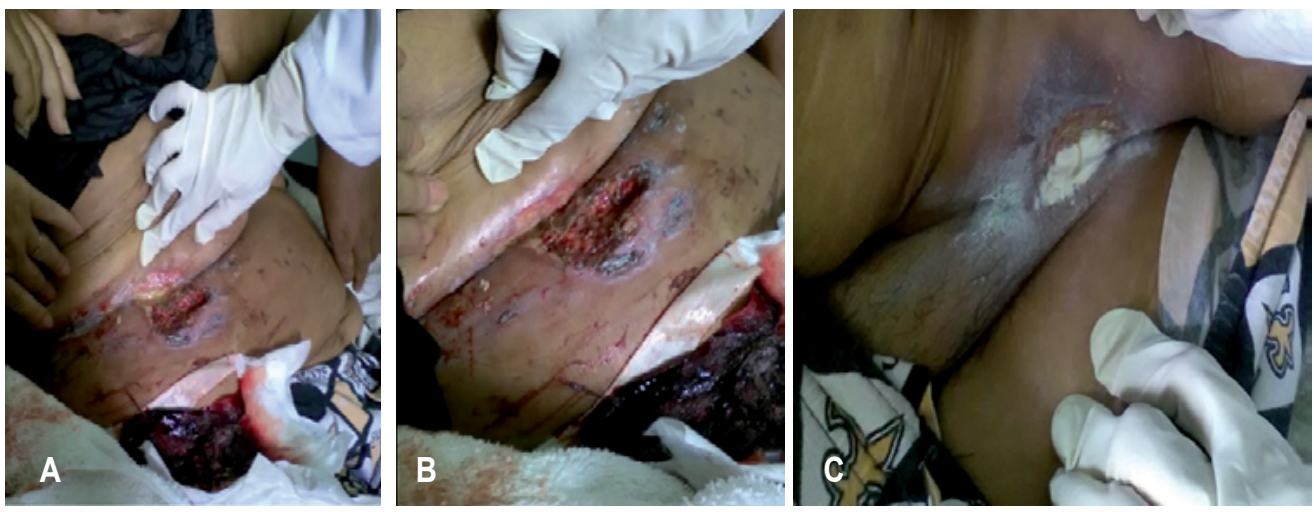

Figura 1. Paciente con lesiones características de la calcifilaxis. Se demuestran lesión ulcerada en región inframamaria izquierda (A), detalles de lesión ulcerada profunda en abdomen (B) y lesión en abdomen con bordes definidos y supurante (C). 
bifosfonatos tipo alendronato $70 \mathrm{mg}$ cada semana. Se le realizan hemodiálisis diarias cortas de 3 horas, por dolor en región del tórax. Además, se indican sesiones de cámara hiperbárica y paratiroidectomía, las que no se pudo realizar por falta de disponibilidad en el centro asistencial.

La paciente no acudió de forma oportuna a realizar sus hemodiálisis de acuerdo al plan establecido. En Julio del 2017 por falta de mejoría de lesiones en piel, acude a Consulta Externa de Dermatología. Ante la sospecha clínica de tuberculosis cutánea se le realiza biopsia de lesión en piel del abdomen en la cual no se observaron micobacterias. Al realizar la biopsia de la úlcera, el sitio se infecta y presenta supuración, el cultivo de la secreción reporta crecimiento de bacterias y Candida albicans. Interconsultantes del Servicio de infectología consideran contaminación de la muestra por lo que se indica tratamiento conservador con limpieza y antibiótico tópico en crema. En agosto de 2017, ingresa a la Sala de Medicina Interna IHSS por falta de mejoría clínica en lesiones con mayor supuración y fiebre. Se indica vancomicina, ertapenem y fluconazol, con evolución satisfactoria de las lesiones. Fue dada de alta para completar seguimiento por consulta externa.

\section{DISCUSIÓN}

La calcifilaxis es sinónimo de Síndrome Urémico Gangrenoso, enfermedad urémica de pequeños vasos y calcificación distrófica. ${ }^{4,5}$ Se caracteriza por vasculopatía obstructiva, con necrosis isquémica y extensas calcificaciones de la túnica media de las pequeñas a medianas arterias de la piel y depende en gran medida de la expresión del factor de crecimiento de fibroblastos-23. ${ }^{2-4}$

El diagnóstico es clínico, tras examinar los antecedentes patológicos, la exploración física, parámetros metabólicos y biopsia de piel que confirma el diagnóstico. ${ }^{4-5}$ Las pruebas de laboratorio, aunque no son específicas pueden apoyar el diagnóstico ${ }^{6,7}$ (Cuadro No. 1).

Cuadro 1. Resultados de exámenes de laboratorios característicos de la arteriolopatía calcificante urémica. ${ }^{6,7}$

\begin{tabular}{lc}
\hline Parámetro & Niveles característicos \\
\hline Creatinina & Elevada \\
Niveles de nitrógeno sérico (BUN) & Elevada \\
Calcio, fósforo, fosfatasa alcalina & Elevada \\
Paratohormona (PTH) & Elevada \\
Aluminio & Elevado \\
Protrombina, cefalina, anticardiolipina, & Alterados \\
homocisteína, Factor V de Leiding, & \\
anticoagulante lúpico & \\
Fibrinógeno & Elevado \\
Amilasa y lipasa & Elevadas \\
Factor reumatoide & Elevado \\
Crioglobulinemia & Elevado \\
Anticuerpos anti Hepatitis C & Positivos \\
\hline
\end{tabular}

En los estudios de imagen, en las radiografías se observan calcificaciones simples de pequeños vasos y tienen buena sensibilidad. La biopsia cutánea de las lesiones produce el diagnóstico definitivo, a pesar del riesgo de propagación de la úlcera. ${ }^{5}$ Los hallazgos histopatológicos en biopsias de piel demuestran calcificación en la capa media de los vasos arteriales y/o arteriolares del tejido dérmico e hipodérmico de entre 0.02 y $0.6 \mathrm{~mm}$, micro trombos, trombos de fibrina en vasos subcutáneos y la dermis superficial, mientras que la epidermis suprayacente presenta necrosis isquémica en el tejido hipodérmico con calcificación periadipocítica, calcificación de capilares septales y/o paniculitis con necrosis lobulillar e infiltrado inflamatorio mixto., ${ }^{4,57}$ En el caso que se realice biopsia de las lesiones se podrá complicar con ulceración epidérmica, necrosis dérmica, paniculitis lobulillar, necrosis grasa, depósitos de calcio en hipodermis y calcificación arterial de vasos hipodérmicos. ${ }^{8-10}$

En un estudio realizado en Hospital Infanta Cristina en España, se describe caso de mujer de 54 años, con ERC secundaria a glomerulonefritis desde1993. En abril del 2011 inicia tratamiento con paricalcitol por hiperparatiroidismo, previamente en tratamiento con carbonato cálcico por hiperfosforemia, aclaramiento de creatinina $(\mathrm{Cr}) 20 \mathrm{ml} / \mathrm{m}$ y PTH $>2.000 \mathrm{pg} / \mathrm{ml}$. Un año después es remitida y se sospecha calcifilaxia por lesiones nodulares subcutáneas pretibiales, inflamadas y dolorosas, con evolución a úlceras de 5 meses de evolución; mamografía con severas calcificaciones lineales de mamas, que no existían en mamografía previa del 2008; igualmente presentaba arteriolopatia urémica a otros niveles. La función renal deteriorada con $\mathrm{Cr} 5.37$, aclaramiento de creatinina/ orina de $24 \mathrm{~h} 12,3 \mathrm{ml} / \mathrm{m}$ con PTH > $2.000 \mathrm{pg} / \mathrm{ml}, \mathrm{Ca}+9,2 \mathrm{mg} / \mathrm{dl}$ y P+ 6,2 mg/dl; se decidió inicio de hemodiálisis, suspender paricalcitol y quelantes cálcicos pautando tratamiento con cinacalcet, sevelamer, tiosulfato sódico (TSS), antibióticos, opiáceos, y paratiroidectomía. ${ }^{8}$ Similar al tratamiento realizado a la paciente del presente reporte de caso excepto la paratiroidectomía que no está disponible en el IHSS; En la paciente descrita del Hospital Infanta Cristina, se decidió no realizar biopsia de las lesiones en piel por el riesgo de diseminación o infección de las lesiones, al contrario de lo que ocurrió con nuestra paciente que debido a la realización de la biopsia se sobreinfectó y fue necesario hospitalizar para cobertura antibiótica. ${ }^{8,11}$

En relación con el tratamiento, actualmente no existe terapia universal. Es multidisciplinario, que incluye tratamiento conservador o quirúrgico. Es básico la suspensión de la administración de calcio vía oral, parenteral o en diálisis y paratiroidectomía de emergencia. ${ }^{4-8}$ Los pacientes sometidos a paratiroidectomía (75\%) tienen mayor supervivencia, que en aquellos que se someten al desbridamiento local. ${ }^{4}$ Akio y cols, reportan que la terapia con vitamina $D$ produce hipercalcemia secundaria, y empeora la calcifilaxis. ${ }^{12-14}$ La hiperfosfatemia se trata con aglutinantes de fosfato y bifosfonatos no basados en calcio 0 cinacalcet. Chetan y cols, indican que el uso de bifosfonatos resuelve los episodios de dolor en 2 días, y la curación de úlceras en 6 meses, sin embargo, la Administración de Medicamentos y Alimentos de los EE. UU (FDA) prohíbe su uso en los pacientes 
con ERC que presenten una tasa de filtración glomerular menor a $30 \mathrm{ml} / \mathrm{min}^{4,10-14}$

El cinacalcet, es un calcimimético que está aprobado para el tratamiento de hiperparatiroidismo secundario en pacientes con ERC y diálisis; actúa aumentando la sensibilidad de las células receptoras de calcio a las paratiroides, con supresión de la PTH. 4, 10-14

El manejo conservador consiste en desbridamiento del tejido necrótico, apoyo nutricional, corrección bioquímica, antibioticoterapia, bifosfonatos, oxígeno hiperbárico y paratiroidectomía. ${ }^{4-6}$ Éste fue el tratamiento que se indicó a la paciente que se describe en el presente caso clínico. Al inicio hubo mejoría de la sintomatología, pero persistencia de las lesiones en piel, y algunas en las que se realizo biopsia presentaron sobreinfección. El manejo analgésico fue morfina dosis de $5 \mathrm{mg}$ IV post hemodiálisis con mejoría del dolor, pero provocó hipotensión por lo que se disminuyó la dosis a $2.5 \mathrm{mg}$ IV post hemodiálisis con buenos resultados. Se indicó sesiones de oxígeno hiperbárico en la paciente, pero no está disponible en el IHSS Tegucigalpa, al igual que el procedimiento de paratiroidectomía.

La terapia con oxígeno hiperbárico aumenta el aporte de oxígeno a los tejidos, y promueve la curación de lesiones cutáneas, previene el edema y el daño del tejido reperfundido, previene la infección de heridas por toxicidad directa contra Clostridium Sp., actividad bacteriostática contra Escherichia y Pseudomonas Sp; promueve la neoangiogénesis, la proliferación de fibroblastos y su conversión a miofibroblastos y la expresión de colágeno. ${ }^{4}$ Para realizar el manejo antibiótico fue necesario hospitalizar a la paciente por 15 días en sala de Medicina Interna de mujeres del IHSS, donde recibió cobertura con ertapenem, vancomicina y fluconazol, con mejoría de la infección y fue sometida a desbridamiento múltiples por el servicio de cirugía, pero sin cierre de la ulcera. Los bifosfonatos inhiben los osteoclastos y la resorción ósea. En modelos animales han demostrado efectos beneficiosos en la prevención de la calcificación arterial. A raíz de estas últimas observaciones, los bifosfonatos se han introducido recientemente como tratamiento de la calcifilaxis con resultados exitosos. ${ }^{5}$ Independientemente del bifosfonato usado se produce mejoría del dolor, con efecto sobre la movilización de las sales de calcio de los tejidos blandos sin observarse hipocalcemia en alguno de los casos. ${ }^{5}$ Se inició tratamiento en la paciente con alendronato a dosis de $70 \mathrm{mg}$ cada semana. ${ }^{7,9,11}$

El tiosulfato de sodio (TSS) es la opción terapéutica parenteral más discutida. Usado desde el 2004 por su efecto vasodi- latador, antioxidante y quelante del calcio, inhibe la precipitación de las sales cálcicas y disuelve los depósitos de calcio ( $\mathrm{Ca}+$ ) depositados en los vasos. Se ha convertido en una promesa farmacológica por su potencial de eficacia y tolerabilidad; ;,9,11,13 Es dializable, y tiene una vida media de 15 minutos con filtrado glomerular conservado ${ }^{7,9} \mathrm{El}$ esquema recomendado es una infusión de 20 a 30 gramos de la solución durante la última hora de la hemodiálisis o poco después de cada sesión de diálisis, 3 veces a la semana. ${ }^{7,9,11,12}$ EI TSS no está disponible en Honduras por lo que no se tienen estudios de experiencias relacionadas en su uso.

En relación a las lesiones en piel no curadas pueden terminar en gangrena cutánea resistente al tratamiento y precisar amputación. Los pacientes con afectación interna pueden desarrollar hemorragia gastrointestinal e infartos de órganos vitales. El pronóstico es malo pues la mayoría de los pacientes fallecen por sepsis cutánea. ${ }^{6,712-15}$ La calcifilaxis conlleva alta morbilidad y mortalidad. La respuesta a cualquier régimen terapéutico parece ser pobre y se asocia con alta mortalidad. El manejo médico conservador está indicado como manejo de elección, en casos de síntomas refractarios, se debe de incluir cámara hiperbárica y manejo quirúrgico con paratiroidectomía. Los factores de mal pronóstico son enfermedad avanzada en el momento del tratamiento y presencia de lesiones isquémicas y necróticas proximales en la piel y tejidos blandos. La isquemia digital tiene un mejor pronóstico que la necrosis en piel proximal. La falta de evidencia en el éxito del tratamiento médico no justifica el nihilismo terapéutico.

Durante el seguimiento en consulta externa de Nefrología en los años 2018-2019, la paciente continuó con las sesiones de hemodiálisis diarias, con analgésicos posterior a hemodiálisis, con quelantes de fósforo, y manejo tópico de lesiones en la piel. Se encuentra estable, en la recuperación de las lesiones en piel.

El tratamiento conservador radica en hemodiálisis diarias, analgésicos, manejo del hiperparatiroidismo terciario y en caso necesario medicación de antibióticos, convirtiéndose en el manejo alternativo al no tener disponibilidad del manejo óptimo como la paratiroidectomía y la cámara hiperbárica.

\section{AGRADECIMIENTOS}

Se reconoce y agradece a la Dra. Mardia Quintanilla, Médico Especialista en Nefrología, institución donde labora, por su apoyo y disposición en la organización del caso clínico.

\section{REFERENCIAS}

1. Rusillo P, Martos Sánchez M, Biechy Baldan M, Liebana Cañada A. Calcifilaxis. Semin Fund Esp Reumatol. 2009;10(4):124-127.

2. Rodríguez-Villarreal I, Callejas R, Sánchez M, Laso N, Gallar P, Ortega $\mathrm{O}$, et al. Calcifilaxis o arteriolopatía urémica calcificante: combinación de tratamientos. Nefro Plus. 2010;3(3):1-48. doi:10.3265/Nefro Plus.pre2010. Jul.10502.

3. Prados-Soler M, Pino M, Fano-López R, Domínguez C. Calcifilaxis severa en paciente en hemodiálisis. Dial Traspl. 2010;31(3):76-78.

4. León Torres K, León Solano C, Rodríguez D. Calcilaxis: Revisión de tema acerca de la calcilaxis del pene. MedLeg Costa Rica. 2016;33 (2):123-132.
5. Torregrosa JV, Durán CE, Barros X, Blasco M, Arias M, Cases A, et al. Tratamiento eficaz de la arteriolopatía urémica calcificante con bifosfonatos. Nefrología. 2012;32(3):275-418. doi:10.3265/Nefrologia.pre2012. Jan.11137.

6. García Villasur P, Arranz Martínez E, Ruiz García A, Angulo Ardoy M. Arteriolopatía urémica calcificante o calcilaxia. SEMERGEN.2007;33(1):31-3.

7. Herrera Muñoz S, Buitrago Villa CA, Serna Toro MJ, Restrepo Valencia CA. Arteriolopatía calcificante urémica, reporte de un caso tratado con tiosulfato de sodio. Rev Colomb Nefrol. 2015;2(1):63-70. doi: 10.22265/ acnef.2.1.198 
8. Ruiz Calero RM, Azevedo LM, Bayo MA, Gonzales B, Cubero JJ. Regresión de calcificaciones vasculares en paciente con calcifilaxia. Nefrología. 2016;36(5):556-81.

9. Navarro JA, Pedraza Cezón LA, De Pablos Martin MP, González Monte E, Polanco Fernández N, Gutiérrez Sánchez J, et al. Tratamiento con tiosulfato de sodio tópico en calcilaxia de paciente con trasplante renal activo. Nefrología. 2016;36(5):556-581.

10. Rodríguez L, Di Martino B, Contreras R, Rodríguez M, Knopfelmacher $\mathrm{O}$, Bolla de Lezcano L, et al. Calcinosis cutis metastásica: calcifilaxis (arteriolopatía urémica calcificada). A propósito de un caso. OurDermatologyonline. 2013;4(3):358-360.

11. Miguel Bouzas JC, Castro Tubío E, Villamayor Blanco L. Tiosulfato sódicoa bajas dosis en el tratamiento de la arteriolopatía urémica calcificante. FarmHosp. 2017;41(5):638 - 639.

12. Latus J, Kimmel H, Ott G, Ting E, Alscher MD, Braun N, et al. Early Stagesof Calciphylaxis: Are Skin Biopsies the Answer. Case Rep Dermatol.2011;3:201-205.doi:10.1159/000333007.

13. Jovanovich A, Chonchol M. Calcic Uremic Arteriolopathy Revisited. J AmSoc Nephrol. 2016;27(11):3233-3235.

14. Bashir SO, Mahmoud A, Omer A, Mohamed D. Penile calcific uremicarteriolopathy occurring postparathyroidectomy in a hemodialysis patient. Saudi J Kidney Dis Transpl.2016;27(6):1265-1269.

15. El Azhary R, Hickson L, McBane R. Calciphylaxis. New Engl J. Med.2018;379(4):397-400.
ABSTRACT. Background: The calcifying uremic arteriolopathy or calciphylaxis is a rare, life-threatening syndrome that affects almost exclusively patients with kidney failure and dialysis, characterized by vascular calcification of small and medium-caliber arteries, with subsequent proliferation, fibrosis, and thrombosis that eventually lead to necrosis and skin ulcers. It is associated with end-stage kidney disease and kidney transplantation, with a prevalence of $1-4 \%$ of patients with chronic kidney failure. Treatment is specialized based on a hyperbaric chamber and parathyroidectomy to induce healing. Description of the clinical case: 42-year-old female, recruited in the nephrology outpatient clinic at the Honduran Social Security Institute in 2017, with a history of arterial hypertension and chronic nephropathy, who underwent a kidney transplant in 1998 which was subsequently failed in a hemodialysis program and conservative management since 2005. The patient developed ecchymotic lesions on the trunk and ulcers at sites of subcutaneous nodules that became superinfected, developing signs of a systemic inflammatory response. Laboratory tests showed hyperphosphatemia, parathormone $3518 \mathrm{pg} / \mathrm{ml}$, calcium-phosphorus product 73.5. Given the lack of surgical management (parathyroidectomy) and hyperbaric chamber in the institution, conservative treatment was established based on daily antibiotics, analgesics, and hemodialysis, which presented an improvement in the clinical picture; however, without resolution of her underlying condition of calciphylaxis. Conclusions: Conservative management in the case of patients with calciphylaxis is an available treatment option with a good response in patients with close follow-up.

Keywords: Vascular calcification, Renal insuficiency, Secondary hyperparathyroidism, Uremia. 